\title{
Internet of Things (IoT) Architecture for Flood Data Management
}

\author{
Azimah Abdul Ghapar, Salman Yussof and Asmidar Abu Bakar \\ Universiti Tenaga Nasional, Malaysia \\ azimah, salman,Asmidar@uniten.edu.my
}

\begin{abstract}
Flood disaster is one of the most frequent natural disasters that occur all around the world. For flood prone areas or countries, flood management is an essential part of their governance. In recent years, there has been an increasing use of Information Technology (IT) to facilitate in flood management. For example, the use of sensors to measure hydrological data such as water level and then transmitting that data over the network has become a common practice in many parts of the world. The same goes with the measurement and transmission of geological and meteorological data related to flood. When combined together, this infrastructure forms an Internet of Things (IoT). This paper provides a review on the research works that utilize IoT for flood data management. The paper then proposed an IoT architecture for flood data management that can serve as the basis for the implementation of IoT infrastructure that collect, transmit and manage flood related data.
\end{abstract}

Keywords: flood, flood data management, Internet of Things (IoT)

\section{Introduction}

Disasters are extreme environmental events that affect all areas in the world. When a disaster happens it will interrupt the essential services such as healthcare, electricity, water, transportation and communication. These interruptions will adversely affect the health, social and economy for the local communities and countries. Among many types of disaster, the flood is common in many countries. According to the World Meteorological Organization (WMO), flooding remains the third biggest disaster in the world [1]. Based on a research conducted by Institute of Environmental Studies, more that $60 \%$ of world cities will be vulnerable to flooding in the next 30 years as a result of the climate changes due to effects of the sea-level rise [2].

Until now, various research works in the area of flood data collection, flood monitoring, flood prediction, flood detection, early warning system and data visualization have been conducted aiming to minimize the impact of flood disaster through early prediction. Recent developments in information and Internet technologies provide a great opportunity to enhance disaster management activities. The Internet is currently evolved in several directions and technologies such as Internet of Things (IoT). Previously it is known as the "Internet of Everything", "machine-to-machine (M2M)", "physical computing" and even "ubiquitous computing". Presently, Internet of Things has become one of the technology that has been used as a study to improve the area of disaster management focusing on flood.

This paper is organized as follows: Section 2 describe the concept of IoT, Section 3 reviews IoT in disaster management, Section 4 provides a summary of IoT in flood data management and Section 5 discussed our proposed IoT architecture for flood data management.

Received (August 10, 2017), Review Result (November 15, 2017), Accepted (November 24, 2017) 


\section{Basic Concept of IoT}

At present, the IoT concept has been widely used in many research areas, including disaster management. IoT term was created by Kevin Ashton in 1999 and he defined IoT as uniquely identifiable connected objects using radio-frequency identification (RFID) technology [3]. Later the definition of IoT become clearer when in 2009, the International Telecommunication Union (ITU) defined IoT as " A global infrastructure for the information society, enabling advances services by interconnecting, physically and virtually, things based on existing and evolving interoperable information and communication technologies" [4]. In 2013, IoT European Research Center (IERC) further define IoT as "A dynamic global network infrastructure with self-configuring capabilities based on standard and and interoperable communication protocols where physical and virtual "things" have identities, physical attributes, and virtual personalities and use intelligent interfaces, and are seamlessly integrated into the information network" [5].

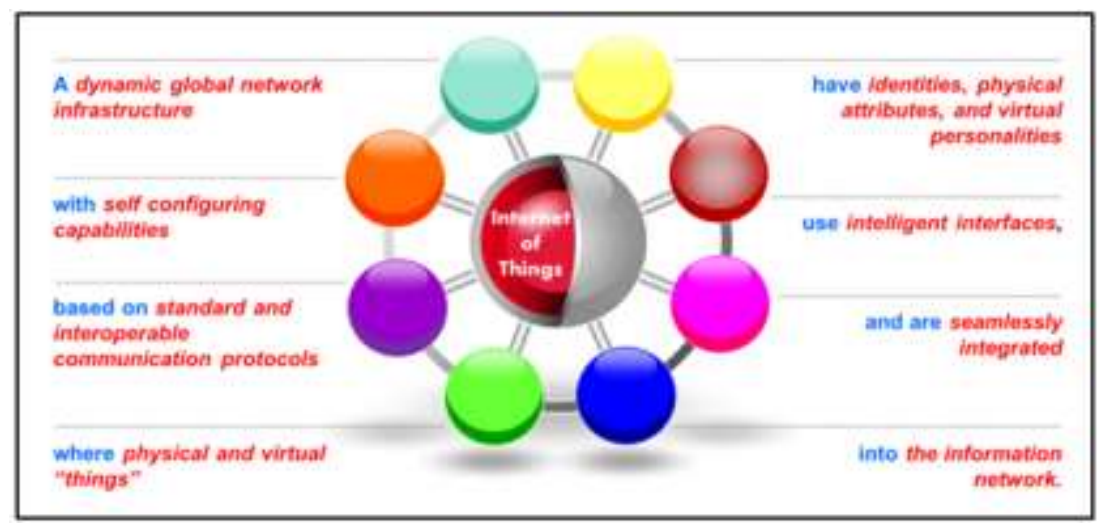

Figure 1. The loT Defined [6]

RFID is a foundational technology for IoT that allows microchips to transfer the identification information through wireless communication. Object that attach to RFID can be identified, monitored and tracked by us via RFID reader. Another technology for IoT is the wireless sensor networks (WSN), which is used to interconnect intelligent sensors. The WSN applications include environmental monitoring, healthcare monitoring, industrial monitoring, and traffic monitoring. These two technologies significantly contribute to the development of IoT. Nowadays many technologies and devices such as barcodes, smart phones, social networks and cloud computing are being used to form an extensive network for supporting IoT as shown in Figure 2.

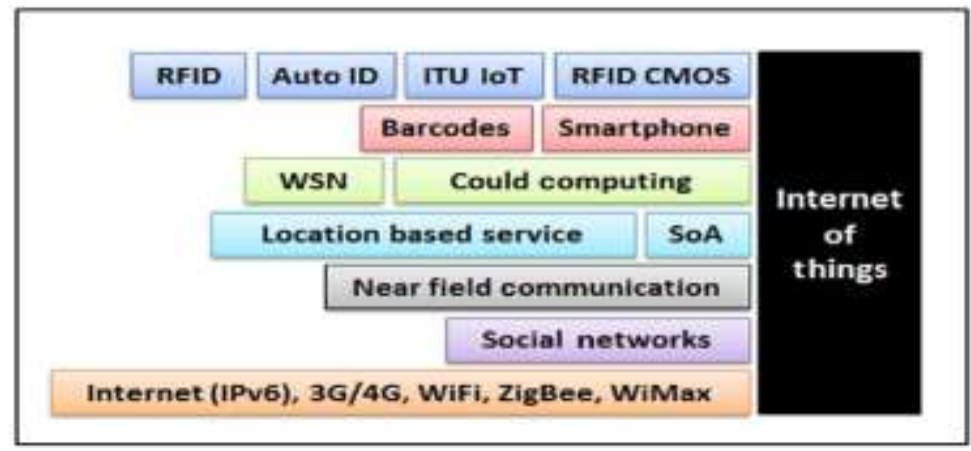

Figure 2. Enabling Technologies for IoT [7] 
The interconnected things and objects obtain the information from the environment and interact with the physical world by using the Internet standard to provide services in information transfer, communications and applications. These objects can be sensors, RFID tags, smart phones, laptops, tablets and embedded system with limited capabilities but able to connect to a wireless or wired network with or without Internet Protocol (IP) addressing [8]. IoT concept has been widely promoted and its application has been widely used in many research areas. Its emergence has become a great strength to facilitate the interaction between things and the environment by using the Internet standard to provide services in information transfer, communications and applications. There are many advantages of incorporating IoT into our lives that can help individuals, businesses and society on a daily basis. The purpose of IoT is to create an environment where the information from "things" can be efficiently shared with others in real-time. Hence by taking advantages of device identification, data collection and sharing capability, data processing and communication, the IoT will make the full use of things to provide services in a more accurate, detailed and in an intelligent manner to all kinds off applications.

Currently, IoT has been applied in many areas including healthcare, safety, manufacturing, smart city, asset tracking and inventory control and environment monitoring. Some of the effective implementation of IoT is in monitoring domestic conditions using ubiquitous sensing system that involves connecting objects to objects. Kelly et al. demonstrate an effective low cost system that offers a flexible solution for condition monitoring and energy management in home [9]. The system can remotely manage and control domestic devices such as electric lamp, water heater, and fridge in providing ambient intelligence to reduce the energy consumption through IoT technology. Yang et al. [10] mentioned that there is a trend in healthcare that moves routine medical checks and other healthcare services from hospital to the home environment. Patient will be monitored by the nurse on duty to check whether the patient is taking medication on time and also to predict for any life-threatening event. The IoT does not only involve connecting objects to objects but also connecting human with objects. The interaction between human and objects has created a smart environment in which the exchange of data and decision is continuous between them and this give assurance that the data used is well monitored. In disaster management, IoT technologies give benefits in terms of monitoring, tracking, controlling and sensing the environment using real time data. IoT devices can also help to offer a simple medium of communication during a disaster.

\section{IoT in Disaster Management}

At present, many research works on IoT in disaster domain have been conducted. This section will provide a summary on research works that implement IoT technologies for addressing natural disasters.

IoT technologies give benefits in terms of monitoring, tracking, controlling and sensing the environment using real time data. Fang et al. introduced the use of IoT to improve environmental monitoring and management tasks [11]. The results from their case study demonstrate that the Integrated Information System (IIS) based on IoT is valuable and efficient for complex tasks in environmental monitoring and management. Ancona et al. highlighted the use of IoT technologies in tackling the complexity in monitoring the flood specifically using rain gauges [12]. IoT provides an interface for data streaming management in real time and at the back end provide data analysis and visualization. In this approach the data collected will be continuously transmitted via the Internet communication infrastructure, to the software components. The software components are designed to compute the stream flow and to quantify the spatial distribution of flood risk for each controlled watershed. Hirabayashi et al. proposed the use of IoT and machine learning based embedded system to predict the probability of floods in a river basin [13]. 
The results of the analysis show a considerable improvement over the currently existing methods. Ke et al. came out with a dynamic monitoring system of fire control with the use of IoT technology which can be useful in the establishment of the information system for fire control system [14]. Perumal et al. proposed an IoT based water monitoring system that measures water level in real-time [15]. If the water level reaches the desired parameter the signal will be fed in real-time to social network like Twitter. Mane et al. developed a flood forecasting system using data mining and wireless sensor network [16]. Their system measures river water level and different weather conditions such as temperature, humidity and vibration through WSN nodes and forecast possibility of future disaster using data mining algorithm.

A warning system is a mechanism that can sense an incoming danger and give warning to the public. Sensing the environment means we monitor the environment for any sign of danger. Technically, an input for a reliable warning system is a result from forecasting process. Forecasting process is a prediction of what will happen based on the data collected. An example, for the flood event, the forecasting process will involve hydrological, meteorological and geological data. These data are collected using devices such as sensors, rain gauges, radar and satellites. Chunquan et al. studied about the use of IoT technologies in monitoring, controlling real time data and came out with an accurate prediction of early warning for urban public safety emergency management [17]. Hu et al. (2011) has proposed an early warning system designed for a tourism crisis in Tibet based on the IoT [18].

As soon as disaster occurs, the traditional telecommunication system, either a landline or a cellular network, may be either partially damaged or entirely damaged by a disaster incident. IoT is a promising technology in helping the rescuers to track the victims and perform coordinated reliefs efforts immediately. Researchers in [19] and [20] in their work highlighted that the IoT-enabled devices that are used to collect the data can offer a minimal device-to-device communication services if the conventional communication infrastructure is out of service during disaster. Researchers in [21] deployed a DfPL (Device free Passive Localisation) WSN in IoT scenario for disaster management which aiming to build upon technology in order to deliver a robust field-trial ready human detection system for disaster situations

Dlodlo had done a study about the potential of using the technologies in making an impact to sustainable environmental monitoring in South Africa [22]. The IoT technologies highlighted covers the area of environmental quality and protection, natural resource management, oceans and coasts management, climate change mitigation and adaptation, biodiversity, conservation and environmental awareness areas. Researchers in [23] have come out with an application framework of IoT in Emergency Management System in China. Chen et al. developed an IoT application for safe building where this application focused on helping people escape from the building in case there is a fire or an earthquake [24].

\section{Motivation towards IoT-Based Flood Data Management}

Flooding is one of the disasters that keep occurring in various part of the world due to the warming climate [13]. According to Aliza et al. [25], various efforts have been taken by flood-prone countries to minimize flood risks including implementing policies and frameworks, building structural and non-structural measures and coordinate the flood authority's activity. - In minimizing the impact of disaster to community, structural and non-structural measures can be implemented. The construction of structural mechanism to control flood such as river improvements, multipurpose dams, underground drainage and tidal gates are examples of structural measures. Non-structural measures involve work related to technology, setting up policies and guideline and humanitarian assistance during flood event. One of the tasks that fall under non-structural measure is the early 
warning system. Early warning system is used to warn the community before the flood takes place. The warning sent is to warn and provide additional time for the community to get ready before the flooding take place. In order to produce a warning, the flood needs to be predicted using flood forecasting system.

Flood forecasting can be defined as the use of real time precipitation and stream flow data in rainfall-runoff and stream flow models to forecast flow rates and water level for periods ranging from a few hours to days ahead which depends on the size of watershed or river basin. Inputs to flood forecasting system include many hydrological, meteorological and geological data collected from an extensive network of monitoring stations. The data includes river level and river flow, rainfall and tide and they originate from various devices [26]. Each of these devices will send data to the server through a communication system. Flood forecasting is needed because it will help to reduce the effects of flood and planning for flood events. The output from flood forecasting will be the input for an early warning system

Various research works have been conducted to support the effort in managing flood. These efforts include the area of flood data collection, flood monitoring, flood prediction, flood detection, early warning system and data visualization and many more with the goals to minimize the impact of flood disasters through early prediction. The current work in flood management involving the Internet of Things can be summarized in the table below:

Table 1. IoT in Flood Management

\begin{tabular}{|c|l|}
\hline Article & \multicolumn{1}{|c|}{ Description } \\
\hline$[13]$ & $\begin{array}{l}\text { Proposed a technique for predicting the occurrence of floods in a river basin } \\
\text { using intelligent IoT system. }\end{array}$ \\
\hline$[15]$ & $\begin{array}{l}\text { Propose a real-time technique for monitoring and measuring water level using } \\
\text { IoT. }\end{array}$ \\
\hline$[16]$ & $\begin{array}{l}\text { Developed a flood forecasting system using data mining and wireless sensor } \\
\text { network. }\end{array}$ \\
\hline$[12]$ & $\begin{array}{l}\text { Proposed flood monitoring using IoT technologies in tackling the complexity } \\
\text { in monitoring the flood specifically using rain gauges }\end{array}$ \\
\hline$[18]$ & $\begin{array}{l}\text { Proposed an early warning system design for a tourism crisis in Tibet based on } \\
\text { the IoT. }\end{array}$ \\
\hline
\end{tabular}

Based on the table above, research works conducted on flood management with IoT technologies are mostly focused on pre-disaster phase, which involves the activities which are taken before a disaster takes place.

\section{Proposed IoT Architecture for Flood Data Management}

Based on the literature review conducted, there has not yet been any IoT architecture proposed for flood data management. Defining an IoT architecture is important because it serves the purpose of conceptualizing the high level structure of the IoT system. The architecture is needed to represent, organize and structure the IoT in a way that enables it to function properly. IoT environment are distributed and heterogeneous. With these criteria it requires the application, hardware, network, software and process involved are capable in supporting devices, services and work flows in IoT environment.

In IoT, the architecture can be viewed as a layered architecture. Each layer is defined by its function and the devices involved in that layer. There are different opinions regarding the number of layers in IoT. However according to researchers in [27] and [28], IoT architecture is generally divided into three layers that are perception, network and application as shown in Figure 3 below. 


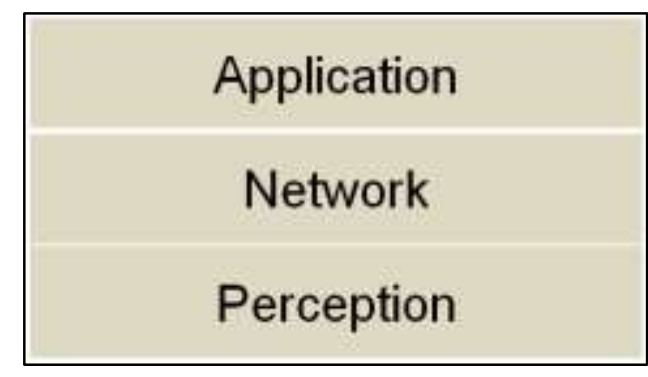

Figure 3. Layers in IoT Generic Architecture

The perception layer is where the data or information originates and it is the core layer of IoT. The purpose of this layer is to acquire the data from the environment with the help of sensors and actuators. This layer detects, collects and processes the data to obtain useful information and transmit it to network layer through the access device. The network layer serves the function of data routing and transmission to the corresponding platforms of upper layer. The information is transmitted via the Internet, mobile communication network and wireless network. The application layer will receive, integrate and store the information. It provides good user interface and specific services depending on the application.

In a pre-disaster phase, early warning system is one of the important efforts to minimize the impact of the disaster to community. In the case of flood, the warning system is sent when the authorities predict the flood will occur based from the output of flood forecasting system. The accuracy of the flood forecasting system depends on many factors. One of the factors is the quality and accuracy of the input data. As mentioned in Section IV above, inputs to flood forecasting system include many hydrological, meteorological and geological data collected from an extensive network of monitoring stations. The data includes river level and river flow, rainfall and tide, and these data originate from various devices [26]. Each of these devices will send data to the server through a communication system. These data is used to forecast a flood. Figure 4 below shows the hydrological data movement from sensors to applications.

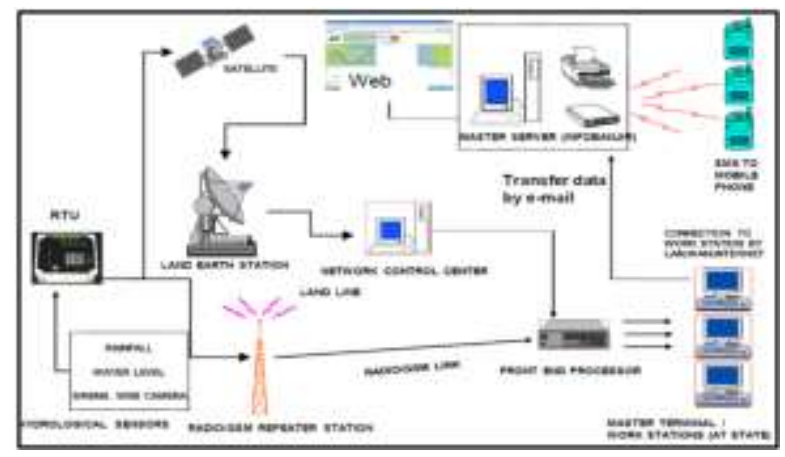

\section{Figure 4. Data Movement from Sensors to Applications}

From the figure, the data movement from sensors to application can be viewed as a series of layered architecture that consists of perception layer, network layer and application layer. The perception layer consists of various devices involved in getting the hydrological, meteorological and geological data. All of these data will be transmitted to the application via mobile communication network, radio access network, wireless sensor network (WSN) and other communications equipment, such as global system for mobile communications (GSM), general packet radio service (GPRS), worldwide interoperability for microwave access (WiMax), 
wireless fidelity (WiFi), Ethernet and Bluetooth. The network layer is responsible for handling this task. The data received by the application layer is stored and passed to the applications that need the data in order to do their tasks. Figure 5 below shows the proposed IoT architecture for flood data management.

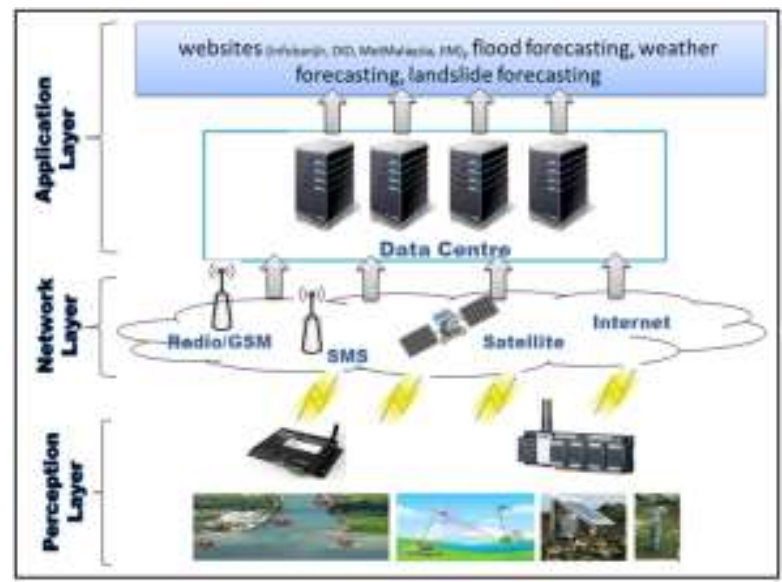

Figure 5. Propose loT Architecture for Flood Data Management

\section{Conclusion}

The success of flood disaster management depends largely on how well flood related data can be collected, managed and utilized. Due to this importance, the use of IoT to facilitate flood data management is seen as a step in the right direction. Many researchers have started to utilize the IoT concept in their work on flood disaster. However, to ensure that all these works that make use of IoT can later be integrated and used together, it is important to have a common architecture that specifies how the different components fit within the larger system and interact with each other. This paper proposed an IoT architecture to serve this purpose. With the proposed architecture, future research works on flood that makes use of IoT will have a reference to specify how the work can fit within the larger flood management system.

\section{References}

[1] "Marufish Disaster Preparedness", Marufish World of Disaster Preparedness, [Online]. Available: marufish.com/disaster/flood/. [Accessed 14 January 2015].

[2] P. J. Ward, "Governance of flood risk management in a time of climate change: the cases of Jakarta and Rotterdam", in Environmental Politics, (2012).

[3] K. Ashton, "That "Internet of Things" thing", RFID Journal, (2009).

[4] ITU-T, "Committed to connecting the world", [Online]. Available: http://www.itu.int/en/ITUT/gsi/iot/Pages/default.aspx. [Accessed August 2017].

[5] IERC, "Internet of Things: Position Paper on Standardization for IoT technologies", EUROPEAN RESEARCH CLUSTER ON THE INTERNET OF THINGS, (2015).

[6] "Coordinating and building a broadly based consensus on the ways to realise the Internet of Things vision in Europe", European Research Cluster on Internet of Things, 2016. [Online]. Available: www.internet-of-things-research.eu/about_iot.htm. [Accessed August 2017].

[7] L. D. Xu, W. He and S. Li, "Internet of Things in Industries: A Survey", in IEEE Transactionals on Industrial Informatics, (2014).

[8] N. E. Petroulakis, I. G. Askoxylakis and T. Tryfonas, "Life-logging in Smart EnvironmentsL Challenges and Security Threats", in Proceedings of the 2012 IEEE International Conference on Communications (ICC), (2012).

[9] S. D. T. Kelly, N. K. Suryadevara and S. C. Mukhopadhyay, "Towards the Implementation of IoT for Environmental Condition Monitoring in Homes", IEEE Sensors Journal, vol. 13, no. 10, (2013), pp. 3846-3853.

[10] G. Yang, L. Xie, M. Mantysalo, X. Zhou, Z. Phang, L. D. Xu, S. Kao-Walter, Q. Chen and L.-R. Zheng, "A Health-IoT Platform Based on the Integration of Intelligent Packaging, Unobtrusive Bio-Sensor, and 
Intelligent Medicine Box”, IEEE Transactions on Industrial Informatics, vol. 10, no. 4, (2014), pp. 2180 $-2191$.

[11] S. Fang, L. D. Xu, Y. Zhu, J. Ahati, H. Pei, J. Yan and Z. Liu, "An Integrated System for Regional Environmental Monitoring and Management Based on Internet of Things", IEEE Transactions on Industrial Informatics, (2014), pp. 1596 - 1605.

[12] M. Ancona, A. Dellacasa, G. Delzanno, A. L. Camera and I. Rellini, "An "Internet of Things" Vision of the Flood Monitoring Problem", in The Fifth International Conference on Ambient Computing, Applications, Services and Technologies, (2015).

[13] Y. Hirabayashi, R. Mahendran, S. Koirala, L. Konoshima, D. Yamazaki, S. Watanabe, H. Kim and S. Kanae, "Global flood risk under climate change", in Nature Climate Change, (2013).

[14] Y. Ke and J. Juncheng, "An approach to the environment of urban building fire safety based on Internet of Things", in Urban Planning and Design Research, (2014).

[15] T. Perumal, M. N. Sulaiman and C. Y. Leong, "Internet of Things (IoT) Enabled Water Monitoring System", in IEEE 4th Global Conference on Consumer Electronics, (2015).

[16] S. S. Mane and M. K. Mokashi, "Real-Time Flash-Flood Monitoring, Alerting and Forecasting System using Data Mining and Wireless Sensor Network", in IEEE ICCSP, (2015).

[17] D. Chunquan and Z. Shunbing, "Research on urban public safety emergency management early warning system based on technologies for the internet of things", Procedia Engineering, vol. 45, (2012), pp. 748754.

[18] W. Hu, X. Wang and A. Kan, "The architecture and design for early warning system of tibet tourism crisis based on the Internet of Things", in IEEE, (2011).

[19] H. Peterson, E. Bacceli, M. Wahlisch, T. C. Schmidt and J. Schiller, "The role of the Internet of Things in Network Resilience", International Internet of Things Summit, (2014), pp. 283-296.

[20] M. Kamaruzzaman, N. I. Sarkar, J. Gutierrez and S. K. Ray, "A study of IoT-based post-disaster management", in International Conference on Information Networking (ICOIN), (2017).

[21] G. Deak, K. Curran, J. Condell, E. Asimakopoulou and N. Bessis, "IoTs (Internet of Things) and DfPL (Device-free Passive Localisation) in a disaster management scenario", Simulation Modelling Practice and Theory, vol. 35, (2013), pp. 86-96.

[22] N. Dlodlo, "Adopting the internet of things technologies in environmental management in South Africa", in Proc. International Conference. Conf. Environment Science Eng, (2012).

[23] Z. Ji and Q. Anwen, "The Application of Internet of Things(IOT) in Emergency Management System in China", in IEEE International Conference Technology Homeland Security, (2010).

[24] T.-Y. Chen, H.-W. Wei, N.-I. Hsu and W.-K. Shih, "A IoT application of safe building in IPv6 network environment", in IEEE 37th Computer Software and Application Conference, (2013).

[25] A. Abdul Latiff and N. H. Arshad, "A Review of Flood Management Governance Framework in Malaysia and Selected Countries", in International Conference on Information Technology and Multimedia (ICIMU), Putrajaya, Malaysia, (2014).

[26] A. Bindal, M. H. Kadhim, D. Parsad and R. B. Patel, "A Pragmatic Review on Algorithmic Approaches for Disaster Management using Wireless Sensor Networks", nternational Journal of Computing and Corporate Research, vol. 4, no. 3, (2014).

[27] Z. Yang, Y. Peng, Y. Yue, W. Xiaobo, Y. Yang and W. Liu, "Study and application on the architecture and key technologies for IOT", in IEEE International Conference on Multimedia Technology (ICMT), (2011).

[28] R. Duan, X. Chen and T. Xing, "A QoS Architecture for IOT”, in IEEE International Conferences on Internet of Things, and Cyber, Physical and Social Computing, (2011). 